$16^{\text {th }}$ International Congress of Metrology, 05002 (2013)

DOI: $10.1051 /$ metrology/201305002

(C) Owned by the authors, published by EDP Sciences, 2013

\title{
EMRP Project HLT 09 - Metrology for radiotherapy using complex radiation fields
}

Jean-Marc Bordy ${ }^{1, a}$, Claus Andersen ${ }^{2}$, Ulrike Ankerhold ${ }^{3}$, Veronique Dedieu ${ }^{4}$, Frank Delaunay ${ }^{1}$, Jacco De Pooter ${ }^{5}$, Jozef Dobrovodsky $^{6}$, Simon Duane ${ }^{7}$ Ralf-Peter Kapsch ${ }^{3}$, Antti Kosunen ${ }^{8}$, Gabor Machula ${ }^{9}$, Hugo Palmans ${ }^{7}$, Maria Pimpinella $^{10}$, Thorsten Schneider ${ }^{3}$ and Jaroslav Solc ${ }^{11}$

${ }^{1}$ CEA, LIST, DM2I, Laboratoire National Henri Becquerel LNE/LNHB, F-91191 Gif sur Yvette, France

2 Danmarks Tekniske Universitet, DTU, Bygning 101, DK-2800 Kongens Lyngby, Denmark

${ }^{3}$ Physikalisch-Technische Bundesanstalt, PTB, Bundesallee 100, D-38116 Braunschweig, Germany

${ }^{4}$ Universté d'Auvergne, UdA,

${ }^{5}$ VSL B.V., Thijsseweg 11, NL-2629 JA Delft, Netherlands

${ }^{6}$ Slovak Institute of Metrology, SMU, Karloveská 63, SK-842 55 Bratislava, Slovakia,

${ }^{7}$ National Physical Laboratory, NPL, GB-TW11 0LW Teddington, Middlesex, United Kingdom,

${ }^{8}$ Radiation and Nuclear Safety Authority, STUK, Laippatie 4, FI-00880 Helsinki, Finland,

${ }^{9}$ Magyar Kereskedelmi Engedelyezesi Hivatal, MKEH, Hatóság 1534 Budapest, P.f.: 919 Hungary

${ }^{10}$ Istituto Nazionale di Metrologia delle Radiazioni Ionizzanti, ENEA - CR Casaccia, Via Anguillarese, 301,00123

Roma, Italy

${ }^{11}$ Cesky Metrologicky Institut Brno, CMI, Okruzni 30, 63800 Brno, Czech Republic

\begin{abstract}
This paper is intended to give information about the on-going work of the MetrExtRT research project concerned with the dosimetric metrology of modern external beam radiotherapy. It started on the $1^{\text {st }}$ of June 2012 for duration of 36 months and covers the study medium- and high-energy X-rays, high-energy electrons, electronic brachytherapy and scanned proton and carbon ion beams. The project concerns all the steps of traceability from the primary standards through to verification of the dose in and around the tumour, namely: developing new primary standards of absorbed dose to water; studying new detectors and improving the knowledge of the characteristics of existing detectors, useable for quality control and in vivo dosimetry, and publishing guide lines of good practice on their use. MetrExtRT gathers 10 partners that are all National Metrology Institutes (NMI) or Designated Institutes (DI) as represented by the list of authors, two Researcher Excellence Grants (REG) for the development of passive gel dosimetry (Université d'Auverge - France) and diamond detectors (Tor Vergata University - Italy) and up to now 12 collaborators from industry, oncology centres and universities.
\end{abstract}

\section{Introduction}

\subsection{Background / current state of the art}

Advances in technology have enabled the introduction of complex forms of radiotherapy for the treatment of cancer, in which dose is delivered in ways that are far removed from established reference dosimetry conditions. While treated volumes can now conform closely to the defined target, so reducing damage to surrounding normal tissue, the accuracy with which the dose is delivered may fall short of the requirements given by ICRU Report 24 (uncertainty of $5 \%$ at the $95 \%$ confidence level on the dose in the target volume).

\subsection{Needs}

\footnotetext{
${ }^{\mathrm{a}}$ Corresponding author: author@email.org
}

Too low a dose in the target volume increases the chance of treatment failure through recurrence of the cancer while too high a dose can result in higher incidence of severe side effects. Lack of traceability to established reference dosimetry and primary standards makes it more difficult to meet the requirement of ICRU Report 24. The main reasons for this are the lack of primary standards of absorbed dose to water, the gap between the standard reference and clinical conditions laying emphasis on the need for quality controls and in vivo dosimetry methods which should be easier to handle and more accurate.

\subsection{Aims}

In order to improve the situation it is necessary to improve all steps of the metrological chain from the 
primary standards through to verification of the dose in and around the tumour, namely: developing new primary standards of absorbed dose to water; studying new detectors and improving the knowledge of the characteristics of existing detectors useable for quality control and in vivo dosimetry; and publishing guide lines of good practice on their use.

\subsection{How to reach the goals}

To achieve these goals, the first stage is to develop new primary standards where a lack of such reference instruments complying with international protocols is identified i.e. for new electronic brachytherapy sources, for medium-energy X-rays, for small size high-energy Xray beams and for hadrons especially for scanned beams. For high-energy X-rays in radiotherapy, the possibility to measure a new integral quantity similar to dose area product is evaluated. Whatever the radiation beams, dosimetric characteristics i.e. directional and energy response, perturbation factors, linearity, homogeneity and spatial resolution depending on the transfer dosimeters and their calibration protocols are also studied to finally propose robust protocol for in vivo dosimetry and quality control and to evaluate the possibility for assessing absorbed dose to water at a point in small beams. The dosimeters that will be studied (calorimeters, ion chambers, silicon diodes, CVD diamond, ESR/Alanine, radio-chromic and storage film, EPID and gel) have been chosen in order to address the need for point, 2D and 3D measurements.

\section{Progress of the work}

\subsection{Work package 1: Medium energy $X$ rays}

The objectives of the first WP are to develop and compare new primary standards of absorbed dose to water for medium $\mathrm{x}$-ray energies. Thus, two new primary standards at $2 \mathrm{~cm}$ depth in water, following the requirement of the IAEA protocol TRS-398 and based on different methods (calorimetry - ENEA and ionometry $\mathrm{MKEH}$ ) are studied. A comparison between these new standards and the ones which already exist (LNHB) and those which will be slightly modified (PTB and VSL) will be performed. Standard radiation qualities will be selected for this comparison and for transferring the reference to the end users. Guidelines for updating the dosimetry protocol based on absorbed dose to water primary standards will be proposed.

The first step in the design and construction of the ENEA calorimeter has been reached [1]. The temperature rise during irradiation has been calculated (see figure 1). The concept of this calorimeter is new; it is a mixed water/graphite calorimeter. The sensitive elements being made from graphite immersed in water at $2 \mathrm{~cm}$ depth.

In order to select the radiation quality for calibration and comparison, two questionnaires were sent electronically, one to the end users and one to the calibration laboratories. A list of the radiation qualities used for radiotherapy was established based on the 39 answers received from the end users. The radiation qualities used for calibration at present are taken from ISO 4037, IEC 61267 and 60731, CCRI, DIN 6809-5 standards. The quality index used to specify the radiation quality is the half value layer (HVL). Figures 2 and 3 give examples of the balance between the calibration and the end users radiation qualities. They show that, in the medium energy range (about 40-120 keV), one can find radiation qualities matching the end user needs.

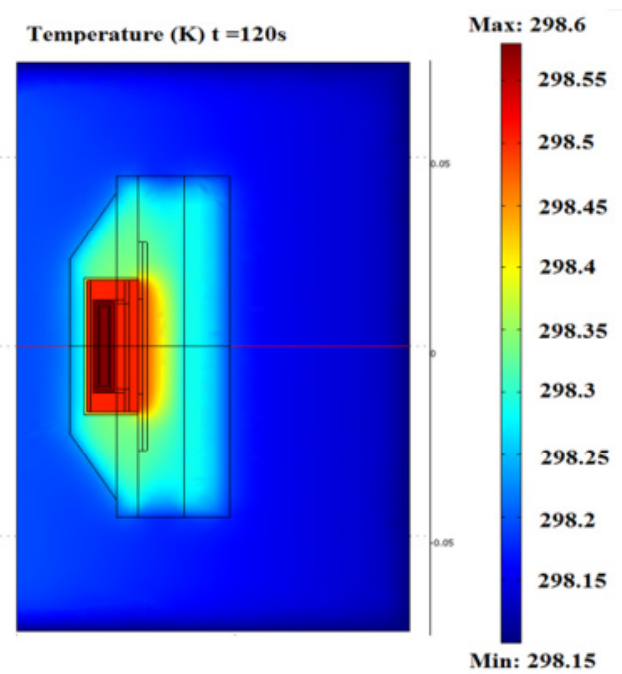

Figure 1. Calculation of the temperature in the ENEA calorimeter after 120 s irradiation.

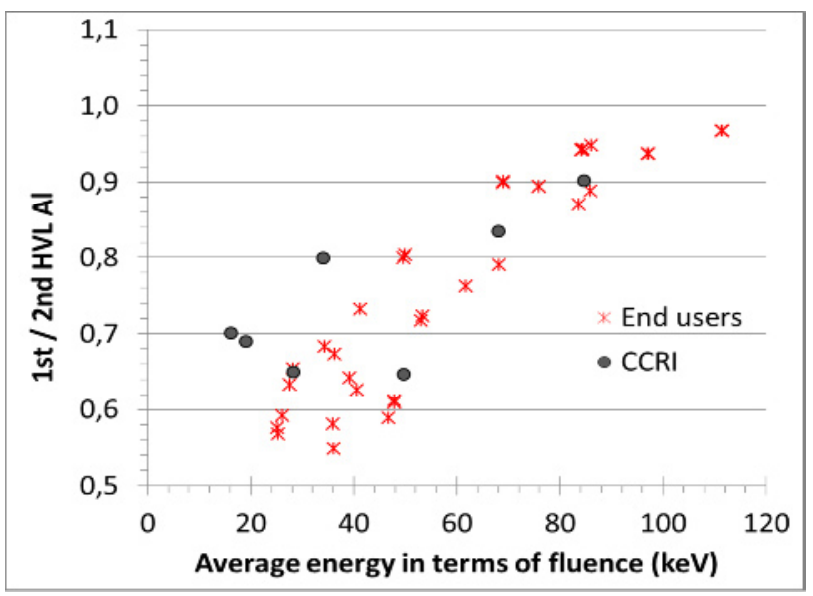

Figure 2. Comparison of the ratios of the first and second HVL for the ends users and CCRI radiation qualities.

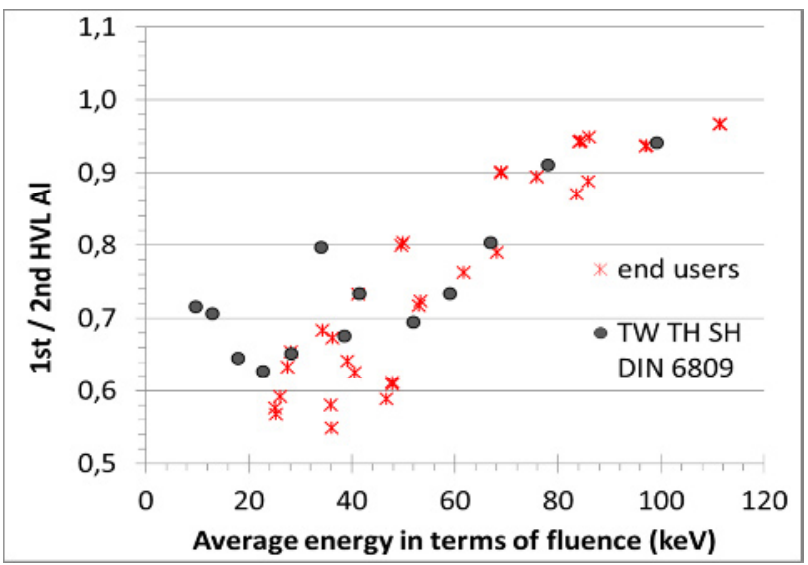

Figure 3. Comparison of the ratios of the first and second HVL for the ends users and DIN6908-5 radiation qualities. 


\subsection{Work package 2: Integral quantity for high energy $X$ rays}

The first part of the work in this WP is described in Dufreinex's paper [2] in this proceeding. It shows the design and construction of the specially designed graphite calorimeter able to be used in small radiation fields (smaller than $2 \times 2 \mathrm{~cm}^{2}$ ) like those used in SRS and SRT (Stereotactic Radio Surgery and Radio Therapy) and IMRT (Imaging Modulated Radio Therapy). The sensitive area of such a calorimeter is larger than the field dimensions so that it measures the energy deposited by the whole radiation beam. The measured quantity is no longer a dose defined at a point but a surface quantity similar to a dose area product. The article also describes the design and first test of two specially designed ionisation chambers having the same area than the calorimeter. Tests are also made to propose a well suited quality index and calibration procedure for this new radiation quantity, finally its future use in treatment planning system software will be evaluated.

\subsection{Work package 3: Hadron therapy}

The aims of this WP are to determine correction factors for both water and graphite calorimeters in scanned proton beams, to compare both types of primary standards in terms of absorbed dose to water, to study by experiment and calculations the correction factors to be applied to ionisation chamber measurements in scanned beams, to study the water equivalence of alanine and radiochromic films and other water equivalent phantom materials in high energy protons beams. To achieve these goals accurate modelling of primary standard and radiation beams are needed. Such models have been produced (figure 4) for the analytical heat transfer simulation in a graphite calorimeter under scanned proton irradiation. This work cannot be done without the support of clinical centres in which experiments will be done, collaborations have been initiated with Clatterbridge Cancer Centre UK (Static $60 \mathrm{MeV}$ Proton Beam), Ružomberok, Slovakia (Scanned $250 \mathrm{MeV}$ Proton Beams), Kern-Fysisch Versneller Instituut Groningen, The Netherlands (Scanned $190 \mathrm{MeV}$ Proton Beam) and Heidelberg Ion Therapy Centre, Germany (Scanned 430/u Carbon Ion Beam).

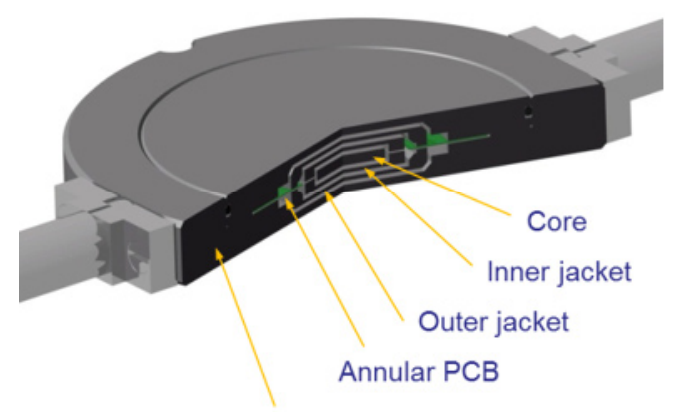

Graphite vacuum body

Figure 4. Model of a graphite calorimeter for the calculation of heat transfer in scanned proton beams.

\subsection{Work package 4: Low energy $X$ rays for brachytherapy}

New low energy micro x-rays generators are now available for improving the efficiency of low energy brachytherapy treatments and avoiding the use of radioactive isotopes. This WP deals with the lack of metrological chain, from the primary standard to the end user's calibration and treatment verification to give recommendations on a calibration chain for this new treatment modality.

The first task, before establishing a primary standard in terms of absorbed dose to water, is to characterize the sources. This can be done through spectrometry and Monte Carlo calculation. In figure 5 the distortion of the spectrum shape due to high photon fluence is evident. Correction of pile up for continuous spectra is done successfully. The Monte Carlo models of the micro xrays generator produced by Carl Zeiss (the so called intrabeam) and the Xoft Axxent tube have been constructed.

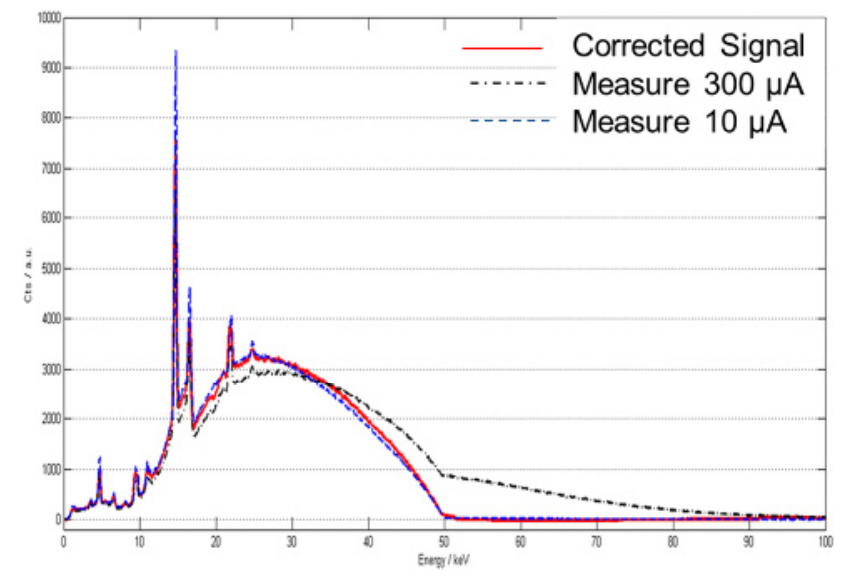

Figure 5. First spectra measured at PTB using an HP GE spectrometer.

\subsection{Work packages 5 and 6: Dosimeter characterisation, quality control and in vivo dosimetry methods}

The aims of WP 5 are to study the use of point like detectors to overcome the lack of primary standards for very small radiation fields. Figure 6 shows how the internal structure of an ionisation chamber can be studied irradiating it partly shielded by lead. The wall and anode influence on the fluence response are visible.

Real time in vivo dosimetry is one of the key issues of radiotherapy because it allows verifications during the treatment and therefore avoids errors. Diamond detectors, already developed at ENEA and Tor Vergata Rome University [3] within the frame work of previous EMRP research program, are optimised in a new configuration suitable for in vivo measurements.

In addition to that work on point detectors, the goal is to characterize 2D (new EBT3 gafchromic film, flexible storage phosphor plates, EPID) and 3D (gels) detectors to be used for verification of complex dose distributions in photon and electron beams. 


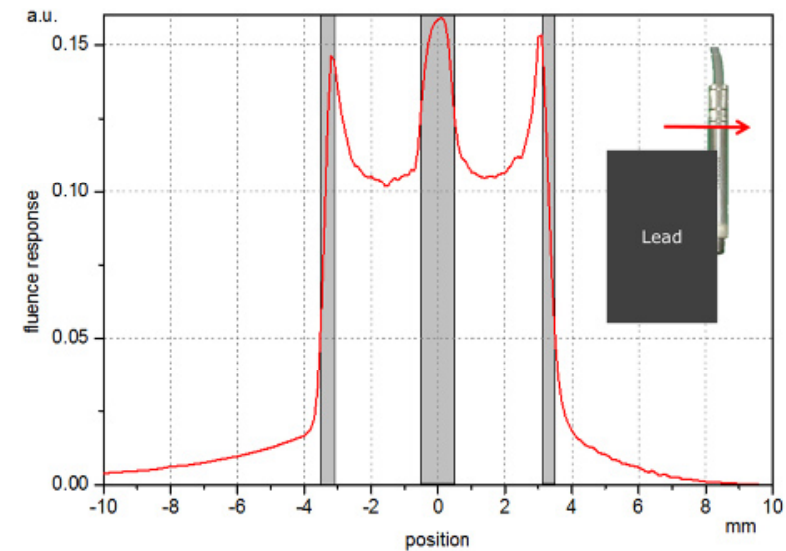

Figure 6. Internal structure of the ion chamber is presented in grey. Measurements achieved with the chamber partly masked by the lead shield are in red. Right top view, to achieve such a red curve the chamber is moved progressively at the edge of the lead shield.

EPID, studied by NPL, are between the 2D and 3D technics because the measurement itself gives 2D information but multiple angle acquisitions and mathematical reconstruction allow the building of $3 \mathrm{D}$ models of the dose distributions inside the patient. As the measurement is made during the treatment the results is a "true" dose distribution which can be used to validate the TPS calculations.

Gel dosimeters are very promising because a single measurement leads to a 3D distribution of doses allowing a direct comparison with treatment planning systems (TPS) calculations. The two main issues are to achieve a high sensitivity as for chemical gel and a good stability after irradiation as for polymer gels. The Université d'Auvergne (UdA) together with LNHB work on an optimized composition by grafting on the gelatin chains specific ligands adapted to bind iron(III) and/or used thickening agents to decrease water mobility and concentration in the gel.

Using the detectors mentioned above, the WP6 is devoted to develop, validate, compare and characterize (in terms of uncertainty budget) measurement methods for the $2 \mathrm{D} / 3 \mathrm{D}$ verification of the treatment planning system. These methods will be based on one dosimeter or the association of several dosimeters. The validation will be done in an anthropomorphic phantom (to be as close as possible to the clinical conditions) on the basis of the comparison of the results of the measurement methods and TPS calculations. Suitable cases for investigation will be chosen among those for which the TPS calculations and the measurements have well known difficulties i.e. where high gradient doses are encountered, such as in dynamic rotational treatment modalities, and in the presence of heterogeneities. Once the methods have been validated a series of recommendations or guidelines will be written and disseminated to the European radiotherapy community. Figure 7 shows an example of an anthropomorphic phantom developed by STUK for breath region. Such a phantom is intended to be scanned for further TPS calculations and detectors will be positioned within it during the simulated treatment for real time measurements of doses.

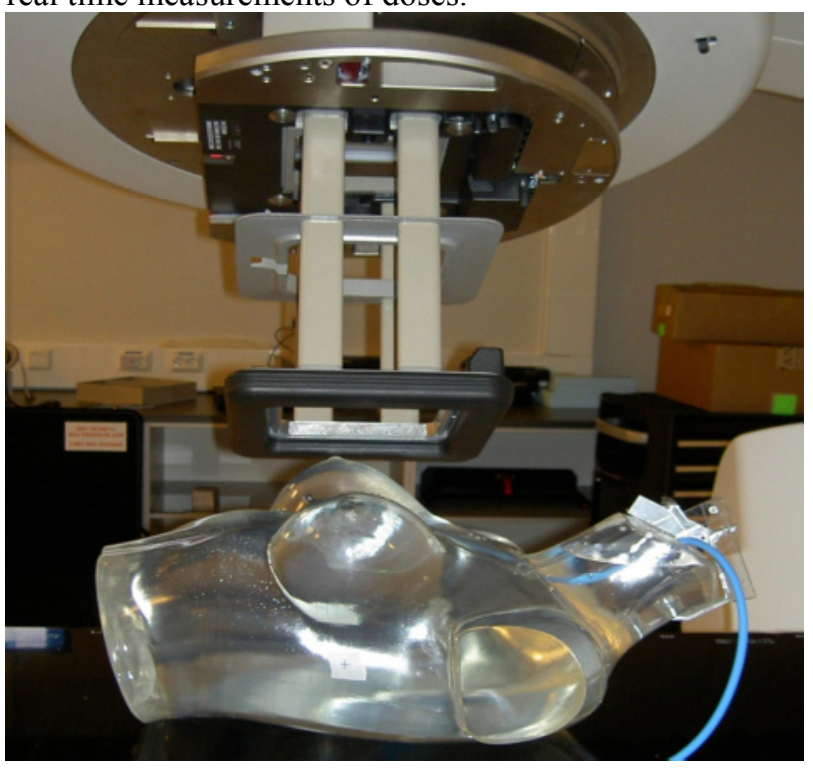

Figure 7. STUK prototype of a female torso phantom, internal organs (lung and bone) and computer control of detector movements inside the phantom will be added.

\section{Conclusions / Impact}

The results of this project will enable international codes of practice for dosimetry in radiotherapy to be updated, producing good practice guidelines for quality control and in vivo dosimetry that will be distributed to the radiotherapy community. By the end of the JRP, the use of absorbed dose to water and its dissemination will be standardized for all types of radiation. The first step towards the application of an integral quantity for very small fields (i.e. establishment of reference, new quality index, secondary standard, and Treatment Planning System -TPS- calculation) will have been completely evaluated. The medical physicists will have more reliable and convenient tools available for quality control and in vivo dosimetry allowing validation of treatment plans. The consequent benefit to cancer patients of this improved dosimetry will come from better treatment outcomes. A workshop will be organised by the end of the JRP in Paris in 2015. This workshop will be open to all the stakeholders.

\section{References}

1. M. Pinto et al., Development of a new in-waterphantom graphite calorimeter for the measurement of absorbed dose to water in medium energy x-ray beams, this proceeding.

2. S. Dufreneix et al., Construction of a large graphite calorimeter for measurements in small fields used in radiotherapy, this proceeding.

3. C Di Venanzio, et al., Characterization of a synthetic single crystal diamond Schottky diode for radiotherapy electron beam dosimetry, February 2013, 40(2), 021712(9 pp), Medical Physics. 